\title{
MATRIX MAPS AND THE ISOMORPHIC STRUCTURE OF BK SPACES
}

\author{
JEFF CONNOR
}

(Communicated by J. J. Uhl, Jr.)

\begin{abstract}
This note gives a characterization of BK spaces that contain isomorphic copies of $c_{0}$ in terms of matrix maps and a sufficient condition for a matrix map from $l_{\infty}$ into a BK space to be a compact operator. The primary tool used in this note is the Bessaga-Pelczynski characterization of Banach spaces which contain isomorphic copies of $c_{0}$. It is shown that weakly compact matrix maps on $l_{\infty}$ are compact and that, if $E$ is a BK space such that there is a matrix $A$ such that $c_{0} \subseteq E_{A}$ and $E_{A}$ is not strongly conull, then $E$ must contain an isomorphic copy of $c_{0}$.
\end{abstract}

\section{INTRODUCTION}

In this note, we give a characterization of Banach-Koordinat (BK) spaces that contain isomorphic copies of $c_{0}$ and a sufficient condition for a matrix map from the bounded sequences into a BK space to be a compact operator. As a corollary, we show that any matrix map from the bounded sequences into a BK space that does not contain an isomorphic copy of $l_{\infty}$ must be compact. These results are obtained by studying the "column space" of a matrix map. As an application, we give an indication of how the isomorphic structure of a BK space affects its "summability" structure.

Before proceeding with the discussion, we pause to collect some definitions and notation. We set

$$
\begin{aligned}
& \omega=\{\text { all scalar sequences }\} \\
& \varphi=\{x \in \omega: x \text { is finitely nonzero }\} \\
& b v=\left\{x \in \omega: \sum_{k=1}^{\infty}\left|x_{k}-x_{k+1}\right|<\infty\right\} \\
& c_{0}=\left\{x \in \omega: \lim _{n} x_{n}=0\right\} \\
& l_{\infty}=\left\{x \in \omega: \sup _{n}\left|x_{n}\right|<\infty\right\} \\
& e^{k}=\text { the } k \text { th unit vector } \\
& e=(1,1,1, \ldots),
\end{aligned}
$$

and, if $E$ is a Banach space, we let $E^{\prime}$ denote its continuous dual and $B_{E}$ denote $E$ 's unit ball. We call the linear functional $p_{n}: \omega \rightarrow \mathbf{C}$, defined by $p_{n}(x)=x_{n}$ for $x=\left(x_{n}\right)_{n=1}^{\infty} \in \omega$, the $n$th coordinate functional.

Received by the editors January $21,1987$.

1980 Mathematics Subject Classification (1985 Revision). Primary 47B37, 46B15.

Key words and phrases. BK space, conull, compact operator, unconditionally convergent. 
Definition. Let $E$ be a Banach space and $\left(x^{n}\right)_{n=1}^{\infty} \subseteq E$. The series $\sum_{n=1}^{\infty} x^{n}$ is unconditionally convergent if any of the following equivalent conditions hold:

(1) $\sum_{n=1}^{\infty} x^{\pi(n)}$ exists for every permutation $\pi$ of $\mathbf{N}$.

(2) $\sum_{k=1}^{\infty} x^{n_{k}}$ exists for every increasing sequence $n_{1}<n_{2}<n_{3}<\cdots$ of positive integers.

(3) $\sum_{k=1}^{\infty} x^{n_{k}}$ exists weakly for every increasing sequence $n_{1}<n_{2}<\cdots$ of positive integers.

(4) $\sum_{n=1}^{\infty} \beta_{n} x^{n}$ for every $\left(\beta_{n}\right) \in l_{\infty}$.

Condition (1) is the usual definition of unconditional convergence. The equivalence of (2) and (3) is the Orlicz-Pettis Theorem. Condition (4) is known as the bounded multiplier test.

Definition. Let $E$ be a Banach space and $\left(x^{n}\right)_{n=1}^{\infty} \subseteq E$. The series $\sum_{n=1}^{\infty} x^{n}$ is weakly unconditionally Cauchy if any of the following equivalent conditions holds:

(1) $\sum_{n=1}^{\infty}\left|f\left(x^{n}\right)\right|<\infty$ for all $f \in E^{\prime}$.

(2) $\sum_{n=1}^{\infty} \alpha_{n} x^{n}$ exists for all $\left(\alpha_{n}\right) \in c_{0}$.

In general, a weakly unconditional Cauchy series is not unconditionally convergent. In fact,

Theorem [Bessaga-Pelczynski]. Let $E$ be a Banach space. $E$ contains a weakly unconditionally Cauchy series which is not unconditionally convergent if and only if $E$ contains an isomorphic copy of $c_{0}$.

(See [D] for a thorough discussion of the convergence of series in Banach spaces.)

We now center our attention on BK spaces. Recall that a Banach space $E$ is a BK space if it is a vector subspace of $\omega$ and $p_{n} \in E^{\prime}$ for all $n \in \mathbf{N} . c_{0}$ and $l_{\infty}$, with their usual norms, are BK spaces. Let $x, y \in \omega$ and $A=\left(a_{n, k}\right)_{n, k=1}^{\infty}$ be an infinite array of scalars. We write $y=A(x)$ if $y_{n}=\sum_{k=1}^{\infty} a_{n, k} x_{k}$ for every $n \in \mathbf{N}$. If $E$ and $F$ are BK spaces and $A(x) \in E$ for every $x \in F$, then $A$ defines a continuous linear map from $F$ into $E$ and we call $A$ a matrix map from $F$ into $E$. If $A\left(B_{F}\right)$ is relatively (weakly) compact in $E$, we say that $A$ is a (weakly) compact map.

If $A=\left(a_{n, k}\right)_{n, k=1}^{\infty}$ defines a matrix map from $F$ into $E$, we let $a^{k}=A\left(e^{k}\right)$ denote the $k$ th column of $A$. Observe that if $\varphi \subseteq F$, then the columns of $A$ are elements of $E$; hence, it makes sense to discuss the convergence of $\sum_{k=1}^{\infty} a^{k}$ in $E$.

For the remainder of the note, we let $E$ and $F$ denote BK spaces and $A$ denote the array $\left(a_{n, k}\right)_{n, k=1}^{\infty}$. Now we are ready to begin. 


\section{WEAKLY COMPACT MATRICES ON $l_{\infty}$ ARE COMPACT}

We start with the key lemma.

Lemma 1. Suppose $A$ defines a matrix map from $l_{\infty}$ into $E$. If $A$ is weakly compact, then $\sum_{k=1}^{\infty} a^{k}$ is unconditionally convergent in $E$.

Proof. We show that $\sum_{k=1}^{\infty} a^{k}$ is weakly subseries convergent and hence, via the Orlicz-Pettis Theorem, unconditionally convergent.

Let $\theta=\left(\theta_{k}\right)$ be an arbitrary sequence of 0 's and 1's, and consider $\sum_{k=1}^{\infty} \theta_{k} a^{k}$. First observe that

$$
\begin{aligned}
C & =\left\{A\left(\sum_{k=1}^{n} \theta_{k} e^{k}\right): n \in \mathbf{N}\right\} \\
& =\left\{\sum_{k=1}^{n} \theta_{k} a^{k}: n \in \mathbf{N}\right\}
\end{aligned}
$$

is a relatively weakly compact set in $E$ since $\left\{\sum_{k=1}^{n} \theta_{k} e^{k}: n \in \mathbf{N}\right\}$ is bounded in $l_{\infty}$. Since $E$ is a $\mathrm{BK}$ space, we have that $E$ 's weak topology and the topology of coordinatewise convergence inherited from $\omega$ are equivalent on the weak closure of $C$ : this follows from the fact that comparable Hausdorff topologies on a compact set are equal. We claim that the coordinatewise limit point, hence the limit point, of $C$ is $A(\theta)$. Observe that $x=\sum_{k=1}^{\infty} \theta_{k} a^{k}$ coordinatewise if and only if $x_{n}=\lim _{\eta} \sum_{k=1}^{\eta} \theta_{k} a_{n, k}$ for all $n \in \mathbf{N}$ since $p_{n}\left(a^{k}\right)=a_{n, k}$. Now, since $\theta \in l_{\infty}, A(\theta)=\left(\sum \theta_{k} a_{n, k}\right)_{n=1}^{\infty}$ is an element of $E$ and $A(\theta)_{n}=\lim _{\eta} \sum_{k=1}^{\eta} \theta_{k} a_{n, k}=\sum_{k=1}^{\infty} \theta_{k} a_{n, k}$, it follows that $A(\theta)$ is the coordinatewise, hence weak, limit point of $C$. Note that the use of the word "the" is proper in that $C$ can only have one coordinatewise limit point. Since $\theta$ was arbitrary, we now have that $\sum_{k=1}^{\infty} a^{k}$ is weakly subseries convergent and hence unconditionally convergent.

Lemma 2. If $\sum_{k=1}^{\infty} a^{k}$ is unconditionally convergent in $E$, then $A$ defines $a$ matrix map from $l_{\infty}$ into $E$ and $A(\beta)=\sum_{k=1}^{\infty} \beta_{k} a^{k}$ for every $\beta=\left(\beta_{k}\right)_{k=1}^{\infty} \in$ $l_{\infty}$

Proof. Let $\beta=\left(\beta_{k}\right) \in l_{\infty}$. First observe that $\sum \beta_{k} a^{k}$ converges since $\sum a^{k}$ is unconditionally convergent, and note that

$$
p_{n}\left(\sum_{k=1}^{\infty} \beta_{k} a^{k}\right)=\sum_{k=1}^{\infty} a_{n, k} \beta_{k}=p_{n}(A(\beta))
$$

for every $n \in \mathbf{N}$. Now, since $\left\{p_{n}\right\}_{n=1}^{\infty}$ is total over $E$, it follows that $A(\beta)=$ $\sum \beta_{k} a^{k}$.

Since $\beta$ was arbitrary, we also have that $A(\beta) \in E$ for every $\beta \in l_{\infty}$, hence $A$ defines a matrix map from $l_{\infty}$ into $E$.

Theorem 3. If $A: l_{\infty} \rightarrow E$ is a weakly compact matrix map, then $A$ is compact. 
Proof. First note that it suffices to show that, for every $\varepsilon>0$ there is a compact set $K_{\varepsilon}$ in $E$ such that $A\left(B_{l_{\infty}}\right) \subseteq K_{\varepsilon}+\varepsilon B_{E}$. Let $\beta=\left(\beta_{k}\right) \in l_{\infty}$ and recall that, for every $\delta>0$, there is an $N_{\delta}$ such that

$$
\left\|\sum_{k=n}^{\infty} \beta_{k} a^{k}\right\|_{E}<\delta \sup _{k}\left|\beta_{k}\right|
$$

for all $n \geq N_{\delta}$, since $\sum_{k=1}^{\infty} a^{k}$ is unconditionally convergent.

Now suppose that $A\left(B_{l_{\infty}}\right) \subseteq M B_{E}, M>0$, and set $\delta=\varepsilon M^{-1}$. Select $N$ such that $(*)$ is satisfied and let $F_{N}$ be the linear span of $\left\{a^{k}\right\}_{k-1}^{N}$. Note that $K_{\varepsilon}=F_{N} \cap M B_{E}$ is compact since $F_{N}$ is finite dimensional and that

$$
\begin{aligned}
A\left(B_{l_{\infty}}\right) & =\left\{\sum_{k=1}^{\infty} \beta_{k} a^{k}:\left(\beta_{k}\right) \in B_{l_{\infty}}\right\} \\
& \subseteq K_{\varepsilon}+\delta M^{-1} B_{E} \\
& =K_{\varepsilon}+\varepsilon B_{E}
\end{aligned}
$$

which completes the proof since $\varepsilon$ was arbitrary.

Rosenthal $[R]$ has shown that any continuous linear operator from $l_{\infty}$ into a Banach space that does not contain an isomorphic copy of $l_{\infty}$ must be weakly compact. This, in tandem with Theorem 3, yields the following corollary.

Corollary 4. Let $E$ be a BK space that does not contain an isomorphic copy of $l_{\infty}$. If $A$ is a matrix map from $l_{\infty}$ into $E$, then $A$ is compact.

\section{THE ISOMORPHIC STRUCTURE OF BK SPACES}

We add one more piece of notation before proceeding. We set

$$
E_{A}=\{x \in \omega: A x \in E\} .
$$

Observe that $A$ is a matrix map from $F$ into $E$ if and only if $F \subseteq E_{A}$ and that Lemma 2 shows that if $\sum_{k=1}^{\infty} a^{k}$ is unconditionally convergent in $E$, then $l_{\infty} \subseteq E_{A}$. In our next lemma, we characterize the matrix maps that take $c_{0}$ into $E$.

Lemma 5. $A$ is a matrix map from $c_{0}$ into $E$ if and only if $\sum_{k=1}^{\infty} a^{k}$ is weakly unconditionally Cauchy in $E$.

Proof. First, suppose that $A$ is a matrix map from $c_{0}$ into $E$. Since $A$ is continuous and $\left(e^{k}\right)_{k=1}^{\infty}$ is a Schauder basis for $c_{0}$, we have that

$$
\begin{aligned}
A(\alpha) & =\operatorname{limit}_{n} A\left(\sum_{k=1}^{n} \alpha_{k} e^{k}\right) \\
& =\operatorname{limit}_{n} \sum_{k=1}^{n} \alpha_{k} A e^{k}=\sum_{k=1}^{\infty} \alpha_{k} a^{k}
\end{aligned}
$$


for any $\alpha=\left(\alpha_{k}\right) \in c_{0}$, hence $\sum_{k=1}^{\infty} a^{k}$ is weakly unconditionally Cauchy in $E$.

Conversely, suppose $\sum a^{k}$ is weakly unconditionally Cauchy in $E$. An argument similar to the one given in Lemma 2 shows that $A(\alpha)=\sum_{k=1}^{\infty} \alpha_{k} a^{k}$ for every $\alpha=\left(\alpha_{k}\right) \in c_{0}$, hence $c_{0} \subseteq E_{A}$ and $A$ defines a matrix map from $c_{0}$ into E.

Theorem 6. $E$ does not contain an isomorphic copy of $c_{0}$ if and only if whenever $A$ is a matrix such that $c_{0} \subseteq E_{A}$ then $l_{\infty} \subseteq E_{A}$ and $A$ defines a compact map from $l_{\infty}$ into $E$.

Proof. Suppose that $E$ does not contain an isomorphic copy of $c_{0}$ and $A$ is a matrix such that $c_{0} \subseteq E_{A}$. Lemma 5 yields that $\sum_{k=1}^{\infty} a^{k}$ is weakly unconditionally Cauchy in $E$ and therefore, thanks to the Bessaga-Pelczynski theorem, unconditionally convergent in $E$. Lemma 2 and the proof of Theorem 3 yield that $l_{\infty} \subseteq E_{A}$ and that $A$ is a compact map from $l_{\infty}$ into $E$.

Conversely, if $\sum_{k=1}^{\infty} a_{k}$ is weakly unconditionally Cauchy in $E$, then the matrix $A$ defined by $a_{n, k}=p_{n}\left(a^{k}\right)$ for all $n, k \in \mathbf{N}$ has the property that $c_{0} \subseteq E_{A}$. Consequently, $l_{\infty} \subseteq E_{A}$ and $A: l_{\infty} \rightarrow E$ is a compact map, which forces $\sum_{k=1}^{\infty} a^{k}$ to be unconditionally convergent in $E$ (Lemma 1). It follows from the Bessaga-Pelczynski Theorem that $E$ cannot contain an isomorphic copy of $c_{0}$.

In the event that $E$ does not contain an isomorphic copy of $l_{\infty}$ (i.e., $E$ is separable), Theorem 6 can be given slightly more succinctly as

Corollary 7. Let $E$ be a BK space that does not contain an isomorphic copy of $l_{\infty}$. E does not contain an isomorphic copy of $c_{0}$ if and only if, whenever $A$ is a matrix such that $c_{0} \subseteq E_{A}$, then $l_{\infty} \subseteq E_{A}$.

This follows immediately from Theorem 6 and Corollary 4 .

\section{SOME APplications to THE SUMmability STRUCTURE OF FK SPACES}

First recall that an FK space is a locally convex Frechet space which is a vector subspace of $\omega$ with continuous coordinate functionals, and that if $E$ is a BK space, then $E_{A}$ can be metrized in such a fashion that it becomes an FK space. An FK space $X$ is said to be strongly conull if $e=\sum_{k=1}^{\infty} e^{k}$ in $X$ 's metric topology. In this section, we restrict our attention to FK spaces of the form $E_{A}$ where $E$ is a $\mathrm{BK}$ space.

We also use the following result in the sequel:

Theorem (Bennett-Sember) [B, S]. Let $E$ be a $B K$ space and $A$ be a matrix. $E_{A}$ is strongly conull if and only if $A: b v \rightarrow E$ is compact.

We can now use Theorem 3 to establish a weak version of a result of Bennett.

Proposition 8. Let $E$ be a $B K$ space that does not contain an isomorphic copy of $l_{\infty}$. If $A$ is a matrix such that $l_{\infty} \subseteq E_{A}$, then $E_{A}$ is strongly conull. 
Proof. Corollary 4 asserts that $A: l_{\infty} \rightarrow E$ is compact. Now, since the inclusion map between FK spaces is continuous and the diagram

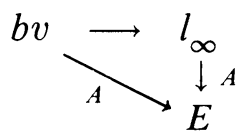

commutes, it follows from the Bennett-Sember result that $E_{A}$ is strongly conull.

Bennett and Kalton [BK] have shown that any separable FK space that contains $l_{\infty}$ setwise is strongly conull. We use Proposition 8 to establish a similar result.

Theorem 9. Let $E$ be a BK space that does not contain an isomorphic copy of $c_{0}$. If $A$ is a matrix such that $c_{0} \subseteq E_{A}$, then $E_{A}$ is strongly conull.

Proof. Since $E$ contains no isomorphic copy of $c_{0}$, we have that $l_{\infty} \subseteq E_{A}$ and that $E$ does not contain an isomorphic copy of $l_{\infty}$. Proposition 8 yields that $E_{A}$ is strongly conull.

Theorem 9 yields some of the "bigness" theorems for conservative weakly sequentially complete spaces (see [W]) since a weakly sequentially complete space cannot contain an isomorphic copy of $c_{0}$.

\section{REFERENCES}

[B] G. Bennett, A new class of sequence spaces, J. Reine Angew. Math. 266 (1974), 49-75.

[BK] G. Bennett and N. J. Kalton, FK spaces containing $c_{0}$, Duke Math. J. 39 (1972), 561-582.

[D] J. Diestel, Sequences and series in Banach spaces, Springer-Verlag, New York and Berlin, 1984.

[R] H. P. Rosenthal, On relatively disjoint families of measures, with some applications to Banach space theory, Studia Math. 37 (1975), 13-36.

[S] J. J. Sember, Summability matrices as compact-like operators, J. London Math. Soc. 2 (1970), 530-534.

[W] A. Wilansky, Summability through functional analysis, North-Holland, Amsterdam, 1984. 